\title{
Dynamic three-dimensional echocardiography combined with semi-automated border detection offers advantages for assessment of resynchronization therapy
}

\author{
Tamas Szili-Torok*, Boudewijn J Krenning, Marco M Voormolen and \\ Jos RTC Roelandt
}

Address: Thoraxcentre, Department of Cardiology, Erasmus MC, Rotterdam, The Netherlands

Email: Tamas Szili-Torok* - t.szilitorok@erasmusmc.nl; Boudewijn J Krenning - b.krenning@erasmusmc.nl; Marco M Voormolen - m.m.voormolen@erasmusmc.nl; Jos RTC Roelandt - j.r.t.c.roelandt@erasmusmc.nl

* Corresponding author

Published: 21 October 2003

Cardiovascular Ultrasound 2003, I:14
Received: 10 July 2003

Accepted: 21 October 2003

This article is available from: http://www.cardiovascularultrasound.com/content/l/I//4

(C) 2003 Szili-Torok et al; licensee BioMed Central Ltd. This is an Open Access article: verbatim copying and redistribution of this article are permitted in all media for any purpose, provided this notice is preserved along with the article's original URL.

\begin{abstract}
Simultaneous electrical stimulation of both ventricles in patients with interventricular conduction disturbance and advanced heart failure improves hemodynamics and results in increased exercise tolerance, quality of life. We have developed a novel technique for the assessment and optimization of resynchronization therapy. Our approach is based on transthoracic dynamic three-dimensional (3D) echocardiography and allows determination of the most delayed contraction site of the left ventricle (LV) together with global LV function data. Our initial results suggest that fast reconstruction of the $L V$ is feasible for the selection of the optimal pacing site and allows identifying LV segments with dyssynchrony.
\end{abstract}

\section{Background}

Simultaneous electrical stimulation of both ventricles in patients with interventricular conduction disturbance and advanced heart failure improves hemodynamics and results in increased exercise tolerance, quality of life [1-3]. Reduction in morbidity and mortality was recently reported.

\section{Case presentation}

We have developed a novel technique for the assessment and optimization of resynchronization therapy. Our approach is based on transthoracic dynamic three-dimensional (3D) echocardiography and allows determination of the most delayed contraction site of the left ventricle (LV) together with global LV function data. We use a selfdeveloped fast rotating transducer, which is connected to a commercially available ultrasound system (GE Vingmed
Vivid FiVe, Horton, Norway). The 64-element phasedarray transducer has a center frequency of $3 \mathrm{MHz}$, second harmonic capabilities and continuously rotates inside the transducer assembly at 8-revolutions/ sec. During resynchronization device implantation, images are obtained with the patient in the left lateral decubitus position with the transducer in the apical position and the image plane rotating around the LV long axis. Images are acquired in sinus rhythm and in two different pacing modes: during right ventricular apical pacing and biventricular pacing. With a self-developed software, using MatLab (The MathWorks, Inc, Natick, MA, USA), the original 2D images are post-processed by placing them in their correct spatial and temporal (ECG reference) position using multi beat data fusion. All the cross-sectional images re-sampled from each dataset are subsequently imported into the TomTec ${ }^{\circledast}$ 4D LV-analysis software (TomTec $^{\circledR}$ Imaging Systems 
GmbH, Germany) for automated endocardial border detection.

Subsequently, the program performs a dynamic surface rendered endocardial reconstruction of the LV in sinus rhythm and in the different pacing modes. For each pacing mode, a time volume curve (TVC) is plotted from which global end-diastolic (LVEDV), end-systolic volumes (LVESV) and ejection fraction (EF) are calculated. The LV endocardial surface is subdivided in 16 segments, which are color-coded for orientation. The difference in time to maximal myocardial contraction between segments is used to assess and measure regional mechanical delay and dyssynergy (Movies 1, 2 and 3 - see additional file 1, additional file 2 and additional file 3 respectively).

Our initial results suggest that fast reconstruction of the $\mathrm{LV}$ is feasible for the selection of the optimal pacing site and allows identifying LV segments with dyssynchrony. Additional hemodynamic evaluation is also possible (Movies 1, 2 and 3). Currently, the LV reconstruction is still off-line. However, real time three-dimensional echocardiography is now available, and the optimal pacing site can be determined on-line.

\section{Conclusions}

Our major finding is that transthoracic dynamic 3D echocardiography performed by a fast rotating transducer and combined with automated contour analysis is feasible to determine mechanical asynchrony during LV contraction. Additionally, it is capable of hemodynamic evaluation and therefore may be advantageous as compared to TDI. Our data suggest that this method may be useful for evaluation and guiding resynchronisation device implantation. Tissue Doppler Imaging (TDI) is the imaging modality, which is currently used to assess the most delayed ventricular site before implantation of a resynchronisation device[4,5]. Although the method is proven to be feasible, it is uncomfortable to use online and, most importantly, assessment of the hemodynamic effect requires additional and separate studies. The aim of our project was to develop and test the feasibility of an alternative approach for determining the optimal pacing site. Our proposed method, based on 3D echocardiographic time-volume curves (TVC), includes additional features such as hemodynamic evaluation by measuring true ventricular volumes. Previous studies demonstrated that generation of TVCs, representing LV volume changes over the cardiac cycle, is feasible and accurate and allows detailed quantitative analysis of LV performance, even in patients with LV dysfunction.

\section{Additional material}

\section{Additional File 1}

Three-dimensional reconstruction of the left ventricle in sinus rhythm (1) The time-volume curves of four different segments are displayed under the three-dimensionally reconstructed ventricles. The endocardial surface is subdivided in 16 color-coded segments. During sinus rhythm, each segment completes myocardial contraction at a different moment, causing left ventricular dyssynchrony. Right ventricular pacing significantly changes the activation pattern, but asynchrony is still present. The right ventricular apical segments contract early, at the site of the position of the right ventricular pacing lead. The largest delay is in the lateral wall in both sinus rhythm and right ventricular apical pacing. During biventricular pacing, optimal mechanical synchrony is achieved.

Click here for file

[http://www.biomedcentral.com/content/supplementary/1476-

7120-1-14-S1.avi]

\section{Additional File 2}

Three-dimensional reconstruction of the left ventricle in, right ventricular $(R V)$ pacing. For more information please see the text for additional file 1 Click here for file

[http://www.biomedcentral.com/content/supplementary/14767120-1-14-S2.avi]

\section{Additional File 3}

Three-dimensional reconstruction of the left ventricle in the biventricular pacing modes. For more information please see the text for additional file 1

Click here for file

[http://www.biomedcentral.com/content/supplementary/1476-

7120-1-14-S3.avi]

\section{References}

I. Daubert JC, Ritter P, Le Breton H, Gras D, Leclercq C, Lazarus A, Mugica J, Mabo $P$ and Cazeau S: Permanent left ventricular pacing with transvenous leads inserted into the coronary veins. Pacing Clin Electrophysiol 1998, 21:239-45.

2. Abraham WT: Cardiac resynchronization therapy for heart failure: biventricular pacing and beyond. Curr Opin Cardiol 2002, 17:346-52.

3. Cazeau S, Leclercq C, Lavergne T, Walker S, Varma C, Linde C, Garrigue S, Kappenberger L, Haywood GA, Santini M, Bailleul $C$ and Daubert JC: Effects of multisite biventricular pacing in patients with heart failure and intraventricular conduction delay. N Engl J Med 200I, 344:873-80.

4. Bax J], Molhoek SG, van Erven L, Voogd PJ, Somer S, Boersma E, Steendijk P, Schalij MJ and Van der Wall EE: Usefulness of myocardial tissue Doppler echocardiography to evaluate left ventricular dyssynchrony before and after biventricular pacing in patients with idiopathic dilated cardiomyopathy. Am J Cardiol 2003, 9 I:94-7.

5. Sogaard P, Egeblad H, Kim WY, Jensen HK, Pedersen AK, Kristensen $\mathrm{BO}$ and Mortensen PT: Tissue Doppler imaging predicts improved systolic performance and reversed left ventricular remodeling during long-term cardiac resynchronization therapy. J Am Coll Cardiol 2002, 40:723-30. 